\title{
My Life as a Psychiatrist: Teacher Is Patients and Their Family, and Treasure Is Colleagues
}

\author{
HEII ARAI* \\ * Department of Psychiatry and Behavioral Science, Juntendo University Graduate School of Medicine, Tokyo, Japan \\ At the retirement from the Juntendo Graduate School of Medicine, I here review my 40-years currier as a \\ psychiatrist and realize that working in this field is very worthwhile and attractive not only for contribution to \\ improving quality of patients' life by treating mental illness but also for making me grow to have a wider view as a \\ human. Accordingly, the patients and their family are my teacher in real-world. Also, I am not sure whether I \\ could complete my term until retirement, if my colleagues were not passionate and supportive as they were. In this \\ sense, my colleagues were my precious treasure in life.
}

Key words: psychiatry, clinical work, patients and family, life events, colleagues

\section{Introduction}

Alzheimer's disease (AD) is the most popular disease (over than 60\%) that causes dementia, but its etiology is still unknown. Accordingly, next-generation drugs for $\mathrm{AD}$, so-called disease-modifying drug, are not yet fully developed and approved through recent strictly-structured (double-blind placebo-controlled) clinical trials. The first case of AD was reported in 1906 by a German psychiatrist, Prof. Alois Alzheimer, and the patient was a presenile female case. Since then, AD had been considered as a kind of presenile dementia 〔early onset in middle age (40-65 years old) ]. But, neuropathological studies support the similarity between $\mathrm{AD}$ and senile dementia, popular dementing disease in older ages. Then, the criteria or definition of $\mathrm{AD}$ come to include not only $\mathrm{AD}$ but also senile dementia. As the lifespan becomes longer, the number of the total $\mathrm{AD}$ patients increases bigger. Now, the problem of $\mathrm{AD}$ is not only medical issue but also social one.
I have been involved in clinical and basic studies on $\mathrm{AD}$ for 40 years. Here, I review my academic currier and would like to add comments on my patients and their families as well as my colleagues with lots of thankfulness.

\section{Progression of $A D$ pathology}

Recently, early-onset $\mathrm{AD}$ or presenile $\mathrm{AD}$ came to be called as "younger patients with AD" (YAD), because it is more easily understood generally. I set up a special out-patient clinic for YAD in 1999 because taking core of YAD patients and their families needed more intensive and comprehensive supports compared to that for old-age AD patients and their families (Table-1).

A recent survey by the Japanese Government disclosed that the most popular disease that causes presenile dementia is vascular dementia, followed by neurodegenerative diseases and head injury. Clinically, presenile dementia which has a big influence on the generation in the prime of life has

\section{Heii Arai}

Department of Psychiatry and Behavioral Science, Juntendo University Graduate School of Medicine

2-1-1 Hongo, Bunkyo-ku, Tokyo 113-0033, Japan

TEL: +81-3-3813-3111 E-mail: heii@juntendo.ac.jp

347th Triannual Meeting of the Juntendo Medical Society: Farewell Lectures of Retiring Professors 〔Held on Mar. 27, 2019〕

〔Received May 10, 2019]〔Accepted May 23, 2019]

Copyright (C) 2019 The Juntendo Medical Society. This is an open access article distributed under the terms of Creative Commons Attribution License (CC BY), which permits unrestricted use, distribution, and reproduction in any medium, provided the original source is properly credited. doi: 10.14789/jmj.2019.65.JMJ19-R08 
Table-1 Our unique/special program for dementia

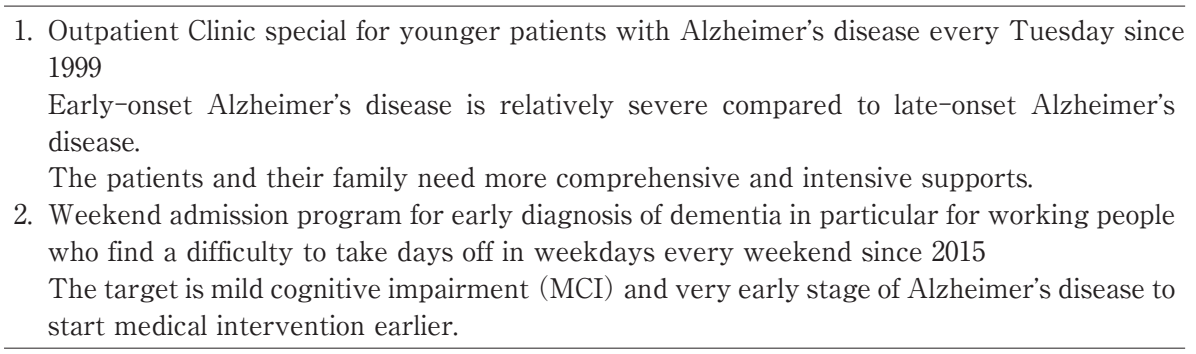

Table-2 Features of early onset dementia

(1) Relatively rapid progression in cognitive decline
(2) Different MRI findings from late onset dementia
(3) More serious psychological and economical influence on the
family
(4) Difficulty to use social capital and care system for late onset
dementia
(5) Importance of disclosure of diagnosis to patient and family
(6) Importance of comprehensive treatment and support
(7) Importance to keep QOL of family

(1) Relatively rapid progression in cognitive decline

(2) Different MRI findings from late onset dementia
family
Difficulty to use social capital and care system for late onset dementia
(6) Importance of comprehensive treatment and support
(7) Importance to keep QOL of family

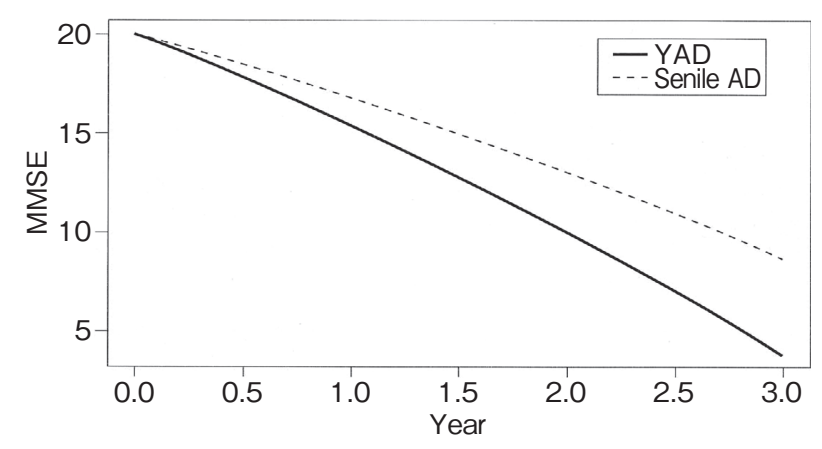

Figure-1 Natural course of YAD and senile AD estimated with initial MMSE score being set at 20.0

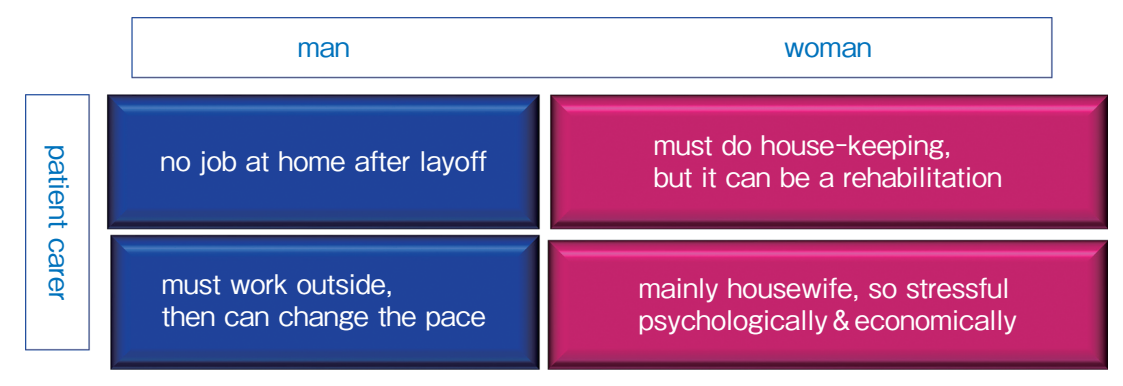

Figure-2 Gender Difference concerning YAD patients' and caregiver's burden

many critical and serious problems (Table-2). First of all, YAD is likely to progress more rapidly in cognitive decline (Figure-1). Gender difference concerning presenile patients' and caregiver's burden was also observed (Figure-2). Dementia after retirement from social activity is little by the meaning as the influence to oneself and the family, but in the case of presenile dementia psychological influence is to the extent it's immeasurable, and isn't the problem of just finishing by drug prescription.

\section{Clinically important issue concerning dementia}

Dementia is not a single disease but a syndrome by which many symptoms gathered focusing on a memory disturbance.

The important first step in clinical work related to dementia is differential diagnosis of dementing illnesses. There is treatable dementia such as normal pressure hydrocephalus, chronic subdural hematoma or hypothyroidism which could be recovered from dementia by appropriate therapy. Depression is also important because it is likely to have "pseudo-dementia" that shows very similar cognitive complaints. Epilepsy is also one of the diseases to be excluded. In this sense, 5 Ds (Dementia, Delirium, Depression, Delusion and Drug-induced psychosis) are most important for differential diagnosis.

In terms of therapeutic intervention, we should pay attention not only to dementia patients but also their families. Also, their circumstances concerning residence or family members should be also important. Thus, the comprehensive supports and 


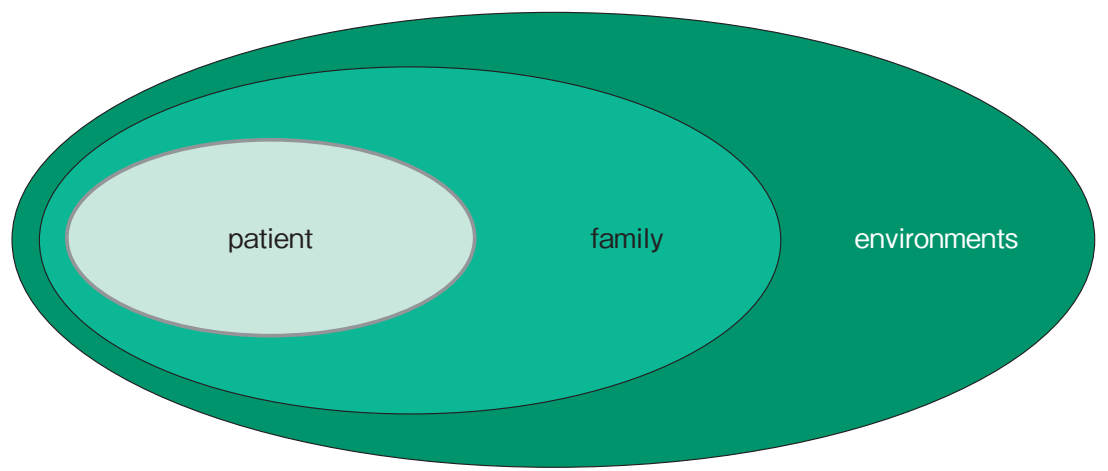

Figure-3 Comprehensive total support is needed for AD
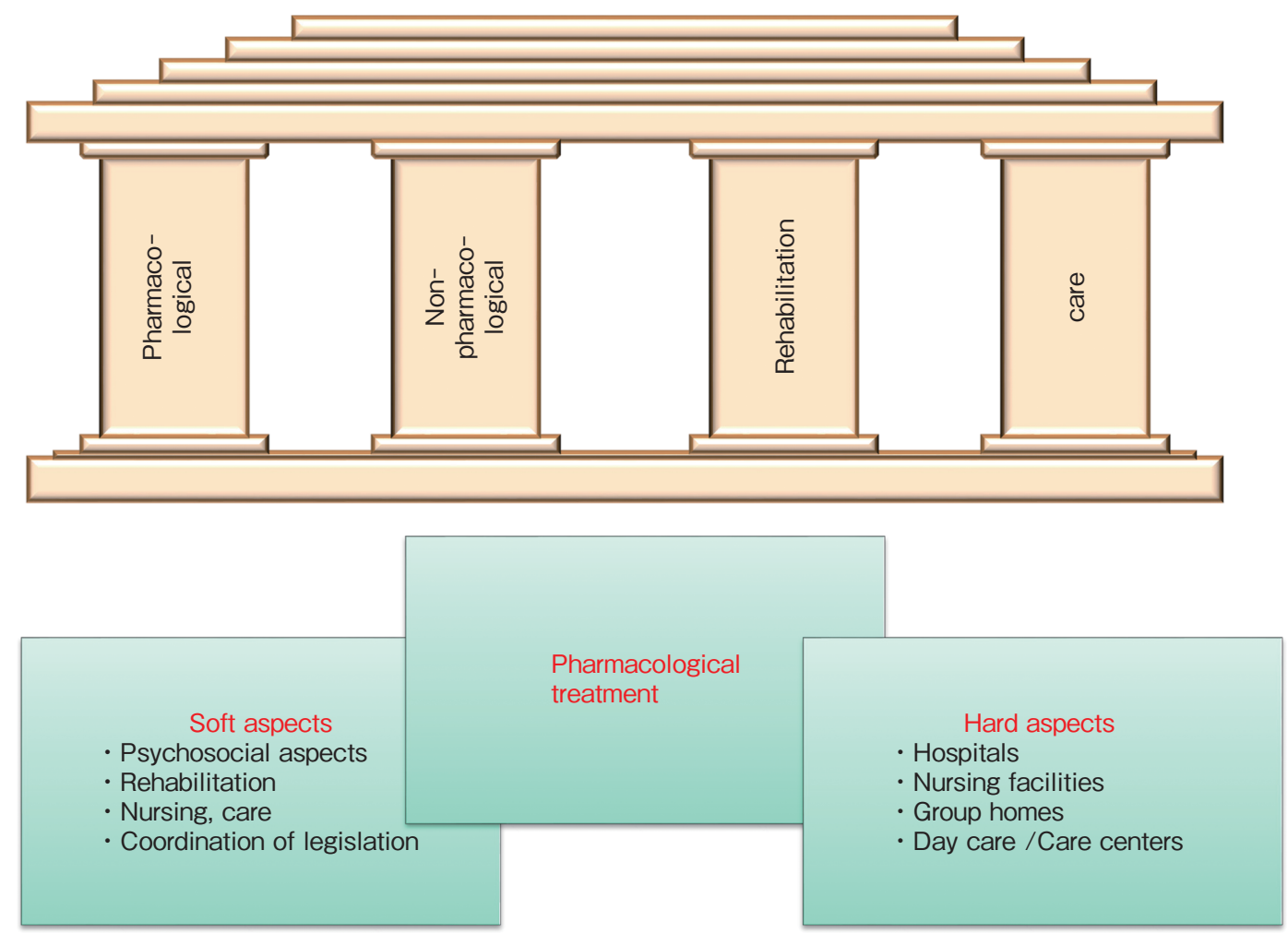

Figure-4 Four major therapeutic strategy for $\mathrm{AD}$

consideration on patients, family and circumstances is necessary (Figure-3). On the other aspect, four important therapeutic strategies such as pharmacological and non-pharmacological approaches, rehabilitation and care should be arranged suitably for each patient (Figure-4).

\section{Are AD patients and their families happy?}

During my clinical currier, I came to raise fundamental questions related to dementia (Figure-5). Those are;
If we had a dementing illness in our lives, can't we become happy?

If dementing illnesses disappeared in the world, can we become happy?

I have been involved in clinical work with dementia patients, especially presenile patients, and I met many very impressive families.

The point is as follows;

Once patient and his/her family accept the presence of YAD, they came to do their best to spend their lives and frequently feel happiness by even small episode such as friend's kind. 
If we had a dementing illness in our lives, can't we become happy ?

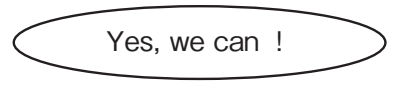

If dementing illnesses disappeared in the world, can we become happy ?

No, we can't !

Once patient and his/her family accept the presence of AD,

they come to do their best to spend their lives and frequently feel

happiness by even small episode such as friend's kindness.

Figure-5 Dementia and happiness

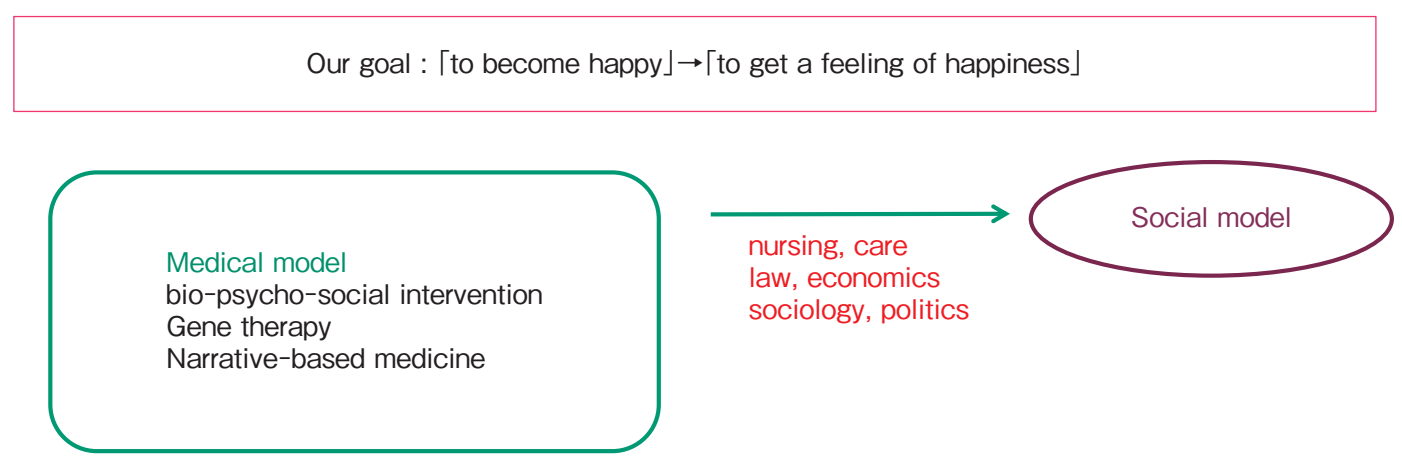

Figure-6 Beyond AD

Such attitude made me realize that I may spend every day without any serious achievements.

Accordingly, I would feel that our goal is not "to become happy (successful life)" but "to get a feeling of happiness in each circumstance". In this sense, we also need to work to establish rather social model to get the goal (Figure-6).

\section{My teachers}

I also came to realize that psychiatry is one of the best opportunities to learn how to spend my life. By listening to many troubles of the patients and their families in my clinic, I noticed that they can objectively view their troubles and learn solutions. In many cases, I also have similar troubles or same conflicts in my private life. The patients and families are relatively serious and hardworking, so they are likely to resolve problems by themselves. Looking back at my life through their life, I could learn the solution and grow myself. Since they frequently gave me hints of solutions for my troubles and concerns, I sincerely feel that patients and their families are teachers in my life. Therefore, I am sure that the answer to a question "Are our clinical works for AD patients and their families?" should be "No".

\section{Research in the Department of Psychiatry, Juntendo University}

The following sentences are quotation from the home page I made for the Department of Psychiatry, Juntendo University.

How do sick people live their lives to the fullest? This is a question that concerns all people and all disciplines of medicine. Particularly in today's advanced and complicated society, this type of question must be addressed, and thus the importance of psychiatry will continue to increase. The core of the clinical training program is the Department of Psychiatry in the Faculty of Medicine and the Graduate School of Psychiatry and Behavioral Sciences. Consistent graduate and postgraduate psychiatry education is provided at seven affiliated hospitals. 


\section{Program contents}

Lectures are roughly divided into systematic lectures for fourth-year medical students and small BSL (Bed Side Learning) group lectures for fifthyear students.

Systematic lectures deal with general and specific topics. General topics include the basic theories of psychiatry and the types and understanding of psychiatric disorders; specific topics thoroughly discuss disease epidemiology, diagnosis, therapy, and prognosis.

In small-group lectures, emergency medicine, drug therapy, psychiatric therapy, test result interpretation and forensic psychiatry are covered in a clinically relevant fashion.

\section{Clinical training}

BSL lasts four weeks. In the mental clinics of four affiliated hospitals, one-on-one training is provided by instructors, with an emphasis on each hospital's specialty: medical psychiatry and consultationliaison psychiatry at the Juntendo Hospital; general psychiatry and community psychiatry at Koshigaya Hospital; consultation-liaison psychiatry at
Urayasu Hospital; and geriatric psychiatry at the Juntendo Tokyo Koto Geriatric Medical Center.

\section{Research}

By reflecting today's society, psychiatry tackles various psychiatric and psychological issues. We have performed various types of research rooted in clinical practice by incorporating psychosocial and molecular biological techniques. In the future, we plan to focus more on a 'team medicine' approach towards our research, and students from various fields, not only medicine, are invited to participate.

Research area in psychiatry has been expanded as the number of treatable clinical disorders has increased along with the development of neuroscience. Based on the wide clinical field, we are trying to integrate the psychopathological studies and neurobiological investigations in order to investigate the pathophysiology and etiology of mental illnesses and to develop new therapies. We are also extending international collaboration projects with foreign research groups, in particular the Cambridge Neurobiology Group (Director: Prof. P.C. Emson, Babraham Insitute, UK).

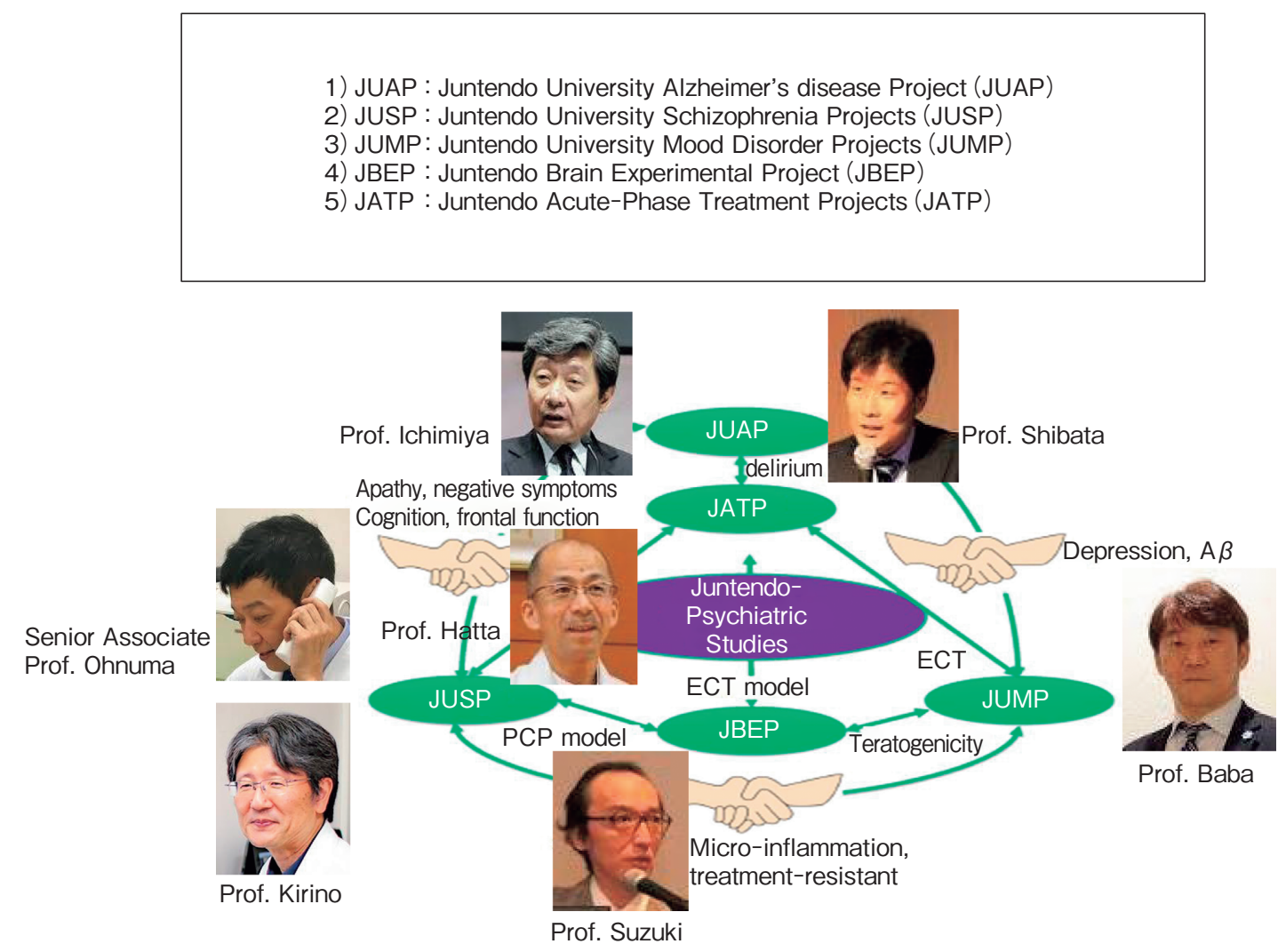

Figure -7 Our projects and leaders 
The following projects are now very actively progressing in our department.

1) JUAP: Juntendo University Alzheimer's disease Project (JUAP) ${ }^{1)}$

2) JUSP: Juntendo University Schizophrenia Projects (JUSP) ${ }^{2)}$

3) JUMP: Juntendo University Mood Disorder Projects (JUMP) ${ }^{3)}$

4) JBEP: Juntendo Brain Experimental Project $(\mathrm{JBEP})^{4)}$

5) JATP: Juntendo Acute-Phase Treatment Projects (JATP) ${ }^{5)}$

These projects are multi-disciplinary and comprehensive study on the pathogenesis and etiology of major psychiatric diseases (Figure-7).

\section{My treasure}

Now, I am sure that all the passionate colleagues of my department during last 22 yeras were the precious treasures for me.

In particular, I would appreciate the project leaders. The leaders of JUAP study are Prof. Y. Ichimiya and Prof. N. Shibata, those of JUSP study are Senior Associate Prof. T. Ohnuma and Prof. E. Kirino, that of JUMP study is Prof. H. Baba, that of JBEP is Prof. T. Suzuki, and that of JATP is Prof. K. Hatta (Figure-7).

I do believe that any basic or clinical study in clinical department of medical school is insignificant and meaningless, unless it is more or less directly related to clinical events or findings to resolve medically from patients' viewpoints. In this sense, therefore, I could not publish a number of papers in psychiatric field without my colleagues' help and support.

\section{Acknowledgements}

At my retirement, I sincerely express my hearty gratitude to the CEO of the Juntendo University, Hideoki Ogawa M.D., Ph.D. for his thoughtful and suggestive guidance up to now.

\section{References}

1) Arai H, Hashimoto N, Sumitomo K, Takase T, Ishii M: Disease state changes and safety of long-term donepezil hydrochloride administration in patients with Alzheimer's disease: Japan-Great Outcome of Longterm trial with Donepezil (J-GOLD). Psychogeriatrics, 2018; 18: 402-411.

2) Ohnuma T, Nishimon S, Takeda M, Sannohe T, Katsuta N, Arai H: Carbonyl Stress and MicroinflammationRelated Molecules as Potential Biomarkers in Schizophrenia. Front Psychiatry, 2018; 9: 82.

3) Ichikawa $T$, Baba H, Maeshima $H$, et al: Serum levels of TDP-43 in late-life patients with depressive episode. J Affect Disord, 2019; 250: 284-288.

4) Ito M, Bolati K, Kinjo $T$, et al: Electroconvulsive stimulation transiently enhances the permeability of the rat blood-brain barrier and induces astrocytic changes. Brain Res Bull, 2017; 128: 92-97.

5) Hatta K, Hasegawa H, Imai A, et al; JAST study group: Real-world effectiveness of antipsychotic monotherapy and polytherapy in 1543 patients with acute-phase schizophrenia. Asian J Psychiatr, 2019; 40: 82-87. 